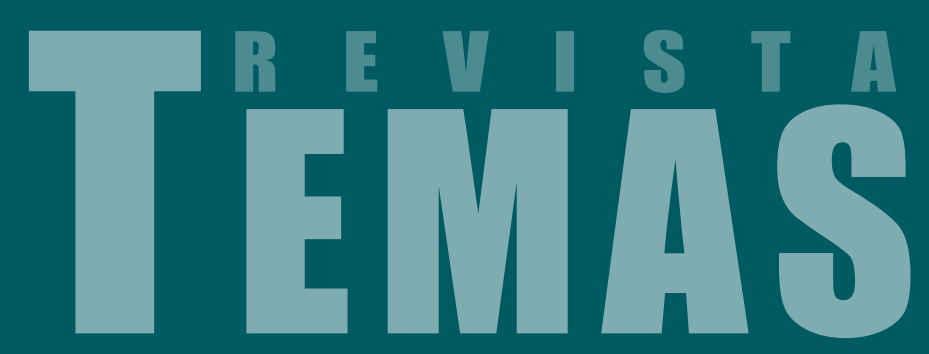

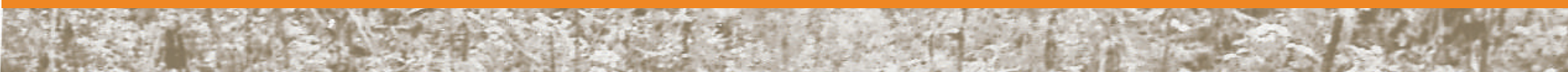

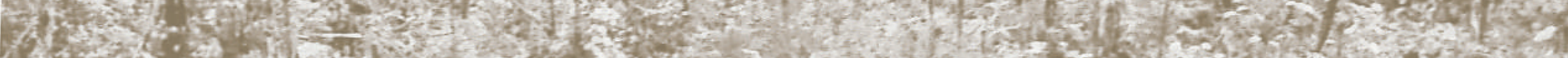

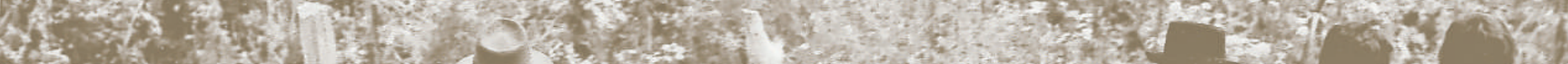

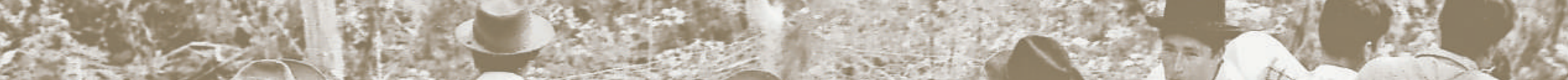

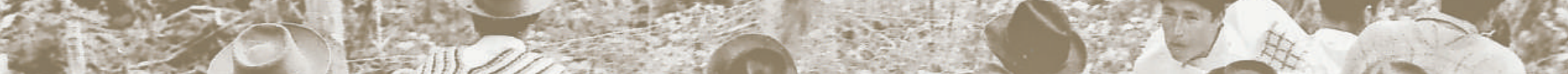

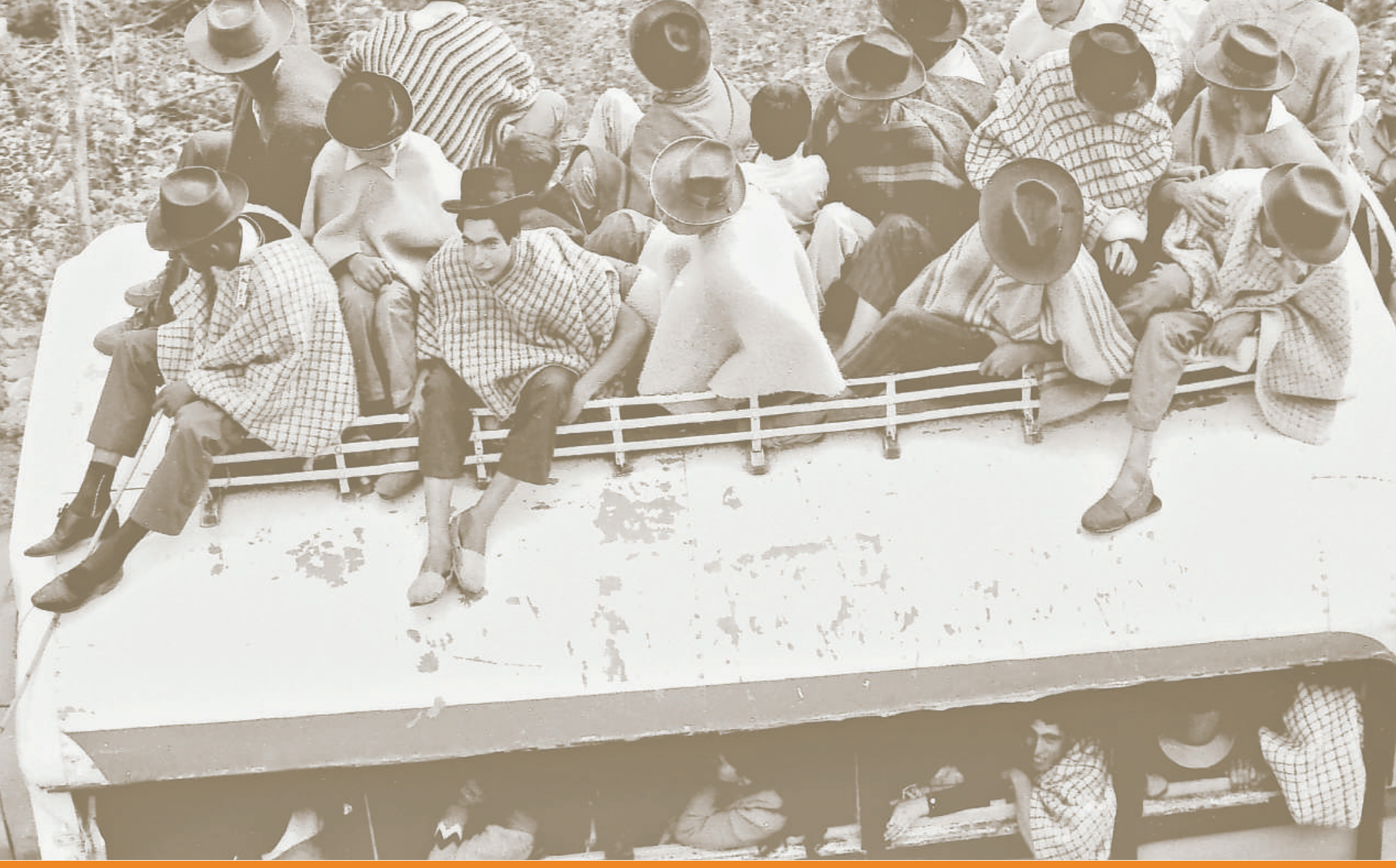





\section{HACIA UNA COMPRENSIÓN DEL ESTADO, PRESENTE EN EL RÉGIMEN DE COALICIÓN BIPARTIDISTA DEL FRENTE NACIONAL EN COLOMBIA [1958 - 1974]'}

(Recepción: Mayo 31 de 2013- Aceptación: Junio 19 de 2013)

Germán Giovanni Báez Plata*

\section{Resumen}

En este artículo se presentan los planteamientos más relevantes frente a la teoría de Estado que se desarrolló en el proceso histórico-político de Colombia en el denominado régimen de coalición bipartidista, durante los años 1958 a 1974, así como el resultado de la democracia consociativa, categoría empleada para denominar situaciones políticas de coalición partidista. Analiza los antecedentes históricos de cara a las ideas políticas en el siglo XX y el resultado social que conlleva todo este engranaje político-histórico.

\section{Palabras clave}

Frente Nacional, Democracia consociativa, Régimen bipartidista, Coalición.

\section{TOWARD AN UNDERSTANDING OF THE STATE, PRESENT IN THE REGIME OF BIPARTISAN COALITION OF THE FRENTE NACIONAL IN COLOMBIA [1958 - 1974]}

\section{Alustract}

In this paper the most relevant approaches against state theory that developed in the historical-political process of Colombia in the so-called bipartisan coalition regime, including in the years of 1958-1974, and the results of democracy consociational category used to describe situations partisan coalition politics. Analyzes the historical background in the face of political ideas in the twentieth century and the social outcome that entails all this political-historical gear.

\section{Keywords}

National Front, Consociational democracy, Regimen Bipartisan, Coalition.

1 Artículo resultado de la investigación correspondiente a la línea de Filosofía Social Bartolomé de las Casas, desarrollada en la Universidad Santo Tomás de Bogotá, para a optar por el título de Magíster en Filosofía Latinoamericana.

* Licenciado en Filosofía y Economía Política, Magíster en Filosofía latinoamericana por la Universidad Santo Tomás, Bogotá, Docente de Humanidades Universidad Santo Tomás, Bucaramanga. giovanniplata@gmail.com 


\section{Introducción}

Una de las dimensiones más persistentes del pensamiento occidental es la filosofía práctica y, dentro de ella, la filosofía política. Desde Platón hasta Habermas, pasando por Aristóteles, Santo Tomás, Maquiavelo, los contractualistas, los utilitaristas, los socialistas y los liberales, diferentes filósofos se ocupan de la dimensión política de la vida humana. Sus preguntas más recurrentes apuntan a develar una pretendida naturaleza social y política del hombre, o a negarla, a establecer la fundamentación ética del orden político, o a negar su existencia, a establecer las fuentes admisibles de la gobernabilidad y la representatividad, a decidir qué es una sociedad justa o qué es un orden político legítimo.

En un sentido general, se puede llamar política a esa actividad humana que, impulsada por valores e intereses, apunta a la conformación o modificación de un orden de poder. Ese orden de poder puede ser institucional o simplemente factual y espontáneo, puede estar y, a menudo lo está, ligado a un orden social, económico o simbólico, o ser un simple orden político institucional. (Senado de la República de Colombia, 1997).

En el discurso filosófico político se puede determinar que, en primer lugar, los pensadores no suelen escribir como ideólogos, sociólogos o politólogos. Se podría decir que escriben sobre la política como filósofos, tampoco existe una manera unívoca de escribir filosóficamente sobre la política. Además, no todos los filósofos que suelen ser estudiados en un curso de filosofía política se vieron a sí mismos como filósofos eminentemente políticos, como si esa fuera una especialidad a la que ellos se hubieran dedicado. Así, no se vieron a sí mismos ni Platón, ni Aristóteles, ni Kant, ni siquiera Hobbes. Ellos fueron filósofos que, al hacer lo que se Ilama filosofía práctica, hicieron filosofía política. Muy pocos filósofos, Maquiavelo sería un caso, se dedicaron casi exclusivamente a lo político. Pese a todo, algo tendrá que decir sobre lo que define el discurso filosófico político y se tiene otra pista que tomar en cuenta a los filósofos mismos y lo que ellos han hecho. Tomar una lista de pensadores que son estudiables en un curso de filosofía política y tratar de ver en qué modo ellos se ocupan de lo político. Se tiene a Platón, Aristóteles, Hobbes, Locke, Campanela, Marx, Maquiavelo, Mariátegui, Horkheimer, Rousseau, Hegel y Marcuse. Todos estos pensadores dicen algo acerca de lo político. Se puede dividir en dos grupos, quienes tienen una vocación más bien normativa y quienes la tienen más bien denunciativa. Es decir, algunos tratan de plantear las bases éticas y/o racionales del orden político justo o del orden político bueno, mientras que los otros, los denunciantes, se especializan más en la crítica del poder político, en develar el poder y sus injusticias. (Ramírez: 2001, p.35).

La teoría política representa en los países que llevaron a cabo su proceso emancipador un eje fundamental en la construcción de un aparato político legal y estructural como es el caso colombiano, donde a través de su historia se construyó atravesando varios momentos, como se señalarán a continuación.

\section{Las ideas políticas en Colombia}

A finales del siglo XIX, comienza a desarrollarse en Colombia, lenta y gradualmente en el seno de la sociedad rural, la modernización capitalista, caracterizada por la forma industrial de la producción, la formación de un mercado interno y de una clase obrera, la centralización y racionalización de la política, la constitución de ciudadanos y consumidores, y la secularización del orden 
social. Este proceso se extiende durante la primera mitad del siglo XX y genera, a su vez, un proceso de desplazamiento de los valores tradicionales y de la religión como factor de cohesión social, por nuevos valores de carácter más laico y moderno. Este proceso produce una serie de tensiones y contradicciones, resultante de su enfrentamiento con las ideas tradicionales dominantes (Rafael M. Carrasquilla, Marco F. Suárez, José V. Concha y Miguel Abadía Méndez, entre otros) y las ideas modernizadoras (Rafael Uribe, Rafael Reyes, Carlos E. Restrepo y Alfonso López Pumarejo, entre otros). Si se la considera desde la perspectiva de las mentalidades, esa tensión se expresa, como afirma José Luis Romero (1987), en la tensión entre la mentalidad rural y la mentalidad urbana, pues [...] los modelos del progresismo, en todas sus formas moderadas, radicales, socialistas-, todos son hijos de la mentalidad urbana. Es la mentalidad de un grupo que, desde que se constituye, aprende a vivir proyectado y no vegetando. A diferencia de los habitantes del mundo rural, inmersos en la rutina cotidiana, la burguesía es la que transforma la vida en un proyecto, y la une a una imagen dinámica de la realidad.

Pero esas actitudes no deben interpretarse solamente como un signo retardatario de oposición al progreso; hay que interpretarlas también como una preocupación legítima por la erosión o pérdida de los lazos de solidaridad y los factores de cohesión social tradicionales, que en las sociedades agrarias están constituidos por los usos y costumbres ancestrales, pero, sobre todo, por los valores y creencias religiosas. Se trata de una mentalidad tradicionalista, por tanto, los conservadores colombianos más tradicionalistas hacían grandes esfuerzos para "reforzar" la religión, expedir una ley de prensa drástica, prevenir al país del contagio liberal y masónico y comunista, según José Luis Romero (1987).

Por tanto, uno de los principios de la ideología conservadora, desde su surgimiento en Europa, es la defensa de la religión. Pensadores conservadores como Bonald, De Maistre, Hegel, Haller y Coleridge, hicieron de la religión "la verdadera piedra de toque del Estado y la sociedad" (Nisbet, 1995. p. 36), y Colombia no fue la excepción.

Una serie de factores históricos maquinales e internacionales produjo el surgimiento de movimientos políticos de izquierda en Colombia durante la década del sesenta del siglo XX. En el plano nacional, el pacto del Frente Nacional entre los dos partidos tradicionales, Liberal y Conservador. En el plano internacional, las revoluciones china y cubana y la decisión de la Unión Soviética de adoptar la transición pacífica al socialismo. $Y$ en los países del tercer mundo, el triunfo de Vietnam, las guerras en África y los movimientos guerrilleros en América Latina. Ningún país de América ha transitado un periplo tan intenso y tan diverso como Colombia, desde una gran variedad de movimientos guerrilleros hasta la creación de un partido unificado de la izquierda en la primera década del siglo XXI. La política de izquierda, se trata de los movimientos y partidos distintos al Partido Liberal y al Partido Conservador que han planteado un cambio más o menos radical sobre la estructura del poder político, la soberanía nacional, el control del Estado, la distribución de las riquezas, las condiciones de vida del pueblo, la correlación internacional de fuerzas, de todas formas opuestos a la hegemonía de los dos partidos tradicionales y enfrentados al sistema tradicional de detentación. 


\begin{tabular}{|c|c|c|c|}
\hline \multicolumn{4}{|c|}{ 1. CRONOLOGÍA DOCUMENTAL DEL FRENTE NACIONAL } \\
\hline FECHA & DOCUMENTO & PARTICIPANTES & CARACTERÍSTICAS \\
\hline $\begin{array}{l}\text { Marzo } 2 \text { de } \\
1956\end{array}$ & $\begin{array}{l}\text { Carta de López } \\
\text { Pumarejo al } \\
\text { directorio Liberal } \\
\text { de Antioquia }\end{array}$ & $\begin{array}{l}\text { Ex presidente López } \\
\text { Pumarejo - Directorio } \\
\text { Liberal de Antioquia }\end{array}$ & $\begin{array}{l}\text { Propone reforma constitucional para establecer gabinete de } \\
\text { coalición con representación proporcional de todos los grupos. } \\
\text { Se sugiere por primera vez un candidato conservador con apoyo } \\
\text { liberal para suceder al general Rojas Pinilla. Primer paso hacia la } \\
\text { constitución de Frente Nacional. }\end{array}$ \\
\hline $\begin{array}{l}\text { Julio } 24 \text { de } \\
1956\end{array}$ & $\begin{array}{l}\text { Declaración } \\
\text { de Benidorm } \\
\text { (España) }\end{array}$ & $\begin{array}{l}\text { Expresidentes Alberto } \\
\text { Lleras Camargo y } \\
\text { Laureano Gómez }\end{array}$ & $\begin{array}{l}\text { Declaración de principios sobre la situación política, la dicta- } \\
\text { dura y el retorno al gobierno civil. Se reafirma la necesidad de } \\
\text { una coalición bipartidista para derrocar al general Rojas Pinilla. } \\
\text { Primer esbozo de un plan para construir una serie de gobiernos } \\
\text { de coalición. }\end{array}$ \\
\hline $\begin{array}{l}\text { Marzo } 20 \text { de } \\
1957\end{array}$ & Pacto de marzo & $\begin{array}{l}\text { Dirección Nacional } \\
\text { Liberal y Dirección } \\
\text { Nacional Conserva- } \\
\text { dora de orientación } \\
\quad \text { ospinista }\end{array}$ & $\begin{array}{l}\text { Rechazo de los partidos a posible reelección del general Rojas } \\
\text { Pinilla. Programa mínimo de acción y oposición a la dictadura. } \\
\text { Ratificación de la necesidad de gobierno de coalición y de } \\
\text { reformas a la Constitución para evitar el sectarismo. Con este } \\
\text { acuerdo se logra integrar al ospinismo a la coalición bipartidista. }\end{array}$ \\
\hline $\begin{array}{l}\text { Julio } 20 \text { de } \\
1957\end{array}$ & $\begin{array}{l}\text { Pacto de Sitges } \\
\text { (España) }\end{array}$ & $\begin{array}{l}\text { Ex presidentes Alberto } \\
\text { Lleras Camargo y } \\
\text { Laureano Gómez }\end{array}$ & $\begin{array}{l}\text { Ya derrocada la dictadura, este acuerdo fija los pasos para } \\
\text { establecer el gobierno de coalición. Propone los mecanismos e } \\
\text { instituciones básicas del Frente Nacional. Desarrolla principios } \\
\text { esbozados en el pacto de Benidorm. La reforma constitucional } \\
\text { propuesta contempla la paridad en el gabinete ministerial y las } \\
\text { corporaciones públicas por un periodo de doce años, al igual } \\
\text { que el establecimiento de la carrera administrativa. Plantea la } \\
\text { necesidad de plebiscito popular para ratificar las reformas cons- } \\
\text { titucionales sugeridas. Reafirma la necesidad de un candidato } \\
\text { nacional para la presidencia de la República. }\end{array}$ \\
\hline $\begin{array}{l}\text { Octubre } 4 \text { de } \\
1957\end{array}$ & $\begin{array}{l}\text { Decreto } 247 \\
\text { de } 1957\end{array}$ & $\begin{array}{l}\text { Junta Militar de } \\
\text { Gobierno }\end{array}$ & $\begin{array}{l}\text { El decreto, recoge la propuesta expresada en el Pacto de Sitges, } \\
\text { convoca a un plebiscito para el } 1^{\circ} \text { de diciembre con el propósito } \\
\text { de someter a consulta popular la reforma constitucional que esta- } \\
\text { blece las instituciones de coalición. La decisión de la Junta Militar } \\
\text { confirma la voluntad del gobierno de acogerse a la propuesta de } \\
\text { Lleras y Gómez para reconstruir la democracia electoral. }\end{array}$ \\
\hline $\begin{array}{l}\text { Noviembre } \\
22 \text { de } 1957\end{array}$ & $\begin{array}{l}\text { Pacto de San } \\
\text { Carlos (acuerdo } \\
\text { de los partidos) }\end{array}$ & $\begin{array}{l}\text { Principales jefes } \\
\text { políticos de los dos } \\
\text { partidos y miembros } \\
\text { de la Junta Militar de } \\
\text { gobierno }\end{array}$ & $\begin{array}{l}\text { Institucionalización de la coalición de los partidos. Respaldo al } \\
\text { plebiscito del primero de diciembre y a la reforma constitucio- } \\
\text { nal. Se logran acuerdos sobre fechas para elecciones de } 1958 \\
\text { y mecanismos básicos de selección del candidato presidencial } \\
\text { de la coalición. Consolidación definitiva del respaldo bipartista } \\
\text { al establecimiento de un gobierno y unas instituciones para } \\
\text { distribuir el poder político. }\end{array}$ \\
\hline $\begin{array}{l}\text { Enero } 30 \text { de } \\
1958\end{array}$ & $\begin{array}{l}\text { Acta de escru- } \\
\text { tinio general } \\
\text { del plebiscito } \\
\text { Nacional }\end{array}$ & $\begin{array}{l}\text { Comité electoral } \\
\text { nacional, presidida por } \\
\text { Mariano Ospina Pérez }\end{array}$ & $\begin{array}{l}\text { Divulgo los resultados definitivos de la consulta plebiscitaria } \\
\text { que fueron favorables al establecimiento de las instituciones } \\
\text { de coalición }\end{array}$ \\
\hline $\begin{array}{l}\text { Marzo } 30 \text { de } \\
1958\end{array}$ & $\begin{array}{l}\text { Carta de Lau- } \\
\text { reano Gómez a } \\
\text { Alberto Lleras } \\
\text { Camargo }\end{array}$ & $\begin{array}{l}\text { Ex Presidentes Laurea- } \\
\text { no Gómez y Alberto } \\
\text { Lleras }\end{array}$ & $\begin{array}{l}\text { Propone la candidatura de Alberto Lleras, condicionada a un } \\
\text { acuerdo que garantice la presidencia de un conservador para } \\
\text { el periodo siguiente }\end{array}$ \\
\hline $\begin{array}{l}\text { Abril } 12 \text { de } \\
1958\end{array}$ & $\begin{array}{l}\text { Propuesta de } \\
\text { la comisión } \\
\text { delegatarios de } \\
\text { la convención } \\
\text { liberal }\end{array}$ & $\begin{array}{l}\text { Darío Eschandia, Luis } \\
\text { López De Mesa, Jorge } \\
\text { Uribe Márquez, Carlos } \\
\text { Lleras Restrepo }\end{array}$ & $\begin{array}{l}\text { Propone de candidatos a un ciudadano relativamente desco- } \\
\text { nocido, y acepta la propuesta de alteración presidencial bajo } \\
\text { la propuesta de alternación presidencial bajo la condición de } \\
\text { extender el Frente Nacional a cuatro periodos de gobierno. }\end{array}$ \\
\hline $\begin{array}{l}\text { Abril } 14 \text { de } \\
1985\end{array}$ & $\begin{array}{l}\text { Insinuación de la } \\
\text { junta parlamenta- } \\
\text { ria conservadora } \\
\text { en respuesta a la } \\
\text { propuesta liberal }\end{array}$ & $\begin{array}{c}\text { Alfredo Arango Jorge } \\
\text { E, Gutiérrez, Francisco } \\
\text { Gómez, Jorge Rosero, } \\
\text { Hugo Escobar }\end{array}$ & $\begin{array}{l}\text { Acepta la propuesta liberal de alternación presidencial y la ex- } \\
\text { tensión del gobierno de coalición a dieciséis años. }\end{array}$ \\
\hline
\end{tabular}




\begin{tabular}{|c|c|c|c|}
\hline $\begin{array}{l}\text { Abril } 19 \\
\text { de } 1958\end{array}$ & $\begin{array}{l}\text { Acuerdo de los } \\
\text { partidos sobre la } \\
\text { reforma } \\
\text { constitucional }\end{array}$ & $\begin{array}{l}\text { Darío Echandía, } \\
\text { Alfredo Arango, } \\
\text { Jorge Uribe, Jorge E. } \\
\text { Gutiérrez. }\end{array}$ & $\begin{array}{l}\text { Se presenta el texto de un artículo único y transitorio para refor- } \\
\text { mar la constitución, mediante el cual se establecía la alternación } \\
\text { en la presidencia de la República por cuatro periodos de gobier- } \\
\text { nos; elaborado por dirigentes de los dos partidos tradicionales }\end{array}$ \\
\hline Julio de 1958 & $\begin{array}{l}\text { Ponencia para } \\
\text { primer debate } \\
\text { en el senado } \\
\text { del proyecto } \\
\text { reformatorio de } \\
\text { la constitución }\end{array}$ & $\begin{array}{l}\text { Edgardo Manotas y } \\
\text { Enrique Sánchez }\end{array}$ & $\begin{array}{l}\text { Ponencia sobre la alternación presidencial, aprobada por el } \\
\text { Senado de la República en la fecha. }\end{array}$ \\
\hline $\begin{array}{l}\text { Agosto } 4 \text { de } \\
1958\end{array}$ & $\begin{array}{l}\text { Ponencia para } \\
\text { primer debate } \\
\text { en el senado } \\
\text { del proyecto } \\
\text { reformatorio de } \\
\text { la constitución }\end{array}$ & $\begin{array}{l}\text { Edgar Manotas, } \\
\text { Hernán Salamanca y } \\
\text { Hernando Carrizosa }\end{array}$ & $\begin{array}{l}\text { Ponencia sobre la alteración presidencial, aprobada por el senado } \\
\text { de la República en la fecha. }\end{array}$ \\
\hline $\begin{array}{l}\text { Agosto } 6 \text { de } \\
1958\end{array}$ & $\begin{array}{l}\text { Ponencia } \\
\text { para primer y } \\
\text { segundo debate } \\
\text { en la Cámara de } \\
\text { representantes } \\
\text { del proyecto } \\
\text { reformatorio de } \\
\text { la constitución }\end{array}$ & $\begin{array}{l}\text { Carlos Galindo y } \\
\text { Enrique Sánchez }\end{array}$ & $\begin{array}{l}\text { Ponencia sobre la alternación presidencial aprobada por la } \\
\text { Cámara de Representantes en la fecha }\end{array}$ \\
\hline
\end{tabular}

Fuente: Tomado de "Nueva Historia de Colombia" Tomo II Historia Política 1946-1986, pg. 189

Cuatro líneas de pensamiento conformaron movimientos de oposición al Frente Nacional desde sus inicios: el liderado por Alfonso López Michelsen, con el Movimiento Revolucionario Liberal (MRL), el de la Alianza Nacional Popular (Anapo), el del Partido Comunista Colombiano y el de los movimientos guerrilleros conformados bajo la inspiración de las revoluciones china (1949), y cubana (1959). El de López Michelsen se conformó porque se había desvirtuado la idea original de unión de los dos partidos tradicionales colombianos. El de la ANAPO, porque unificaba fuerzas políticas alrededor del regreso del general Rojas Pinilla al gobierno. El Partido Comunista porque había sido ilegalizado durante el régimen de Gustavo Rojas Pinilla (1953-1957). Y el de los Movimientos Guerrilleros, porque preconizaban la toma del poder por la fuerza de las armas.

El pacto bipartidista del Frente Nacional no ha sido un fenómeno único en la historia reciente de Colombia: Uruguay y Venezuela en las décadas del 50 y 60, y luego Chile y Argentina en los 80 vivieron algo semejante durante la transición a sus democracias. Jonathan Hartlyn (1993) en su libro La política del Régimen de coalición: La experiencia del Frente Nacional en Colombia, ha denominado a este proceso consociacionalismo para explicar un fenómeno político por el cual las clases dirigentes tradicionales forman amplias coaliciones en momentos de transición hacia un gobierno democrático.

\section{Estado y coalición bipartidista}

El Frente Nacional se constituyó con el argumento de sellar un pacto de paz entre el Partido Liberal y el Partido Conservador, protagonistas de enfrentamientos agudos que siguieron al asesinato del líder liberal Jorge Eliécer Gaitán en 1948. Tomó como punto de partida el propósito de cerrarle el paso a cualquier movimiento de izquierda, especialmente comunista, en un momento de grave enfrentamiento de los más poderosos países de Occidente con la Unión Soviética. Se había roto la alianza de los "Aliados" con la Unión 
Soviética y se había olvidado la simpatía abierta de connotados dirigentes del Partido Conservador con el fascismo, entre otros estaban Silvio Villegas, Gilberto Alzate Avendaño e, inclusive, Laureano Gómez (Galvis y Donadio, 1986). Bastó que la oposición a la reelección del general Gustavo Rojas Pinilla uniera todas las vertientes de los dos partidos para olvidar este confuso pasado y converger en el Frente Nacional. Colombia se alineó de inmediato con los Estados Unidos una vez se rompió la alianza que había llevado a los Aliados al triunfo sobre Alemania.

Colombia ha sufrido, durante más de cincuenta años, los efectos de conflictos armados de distinta índole, que han llevado a que el país sea considerado uno de los más violentos de la tierra; algunos analistas han Ilegado a acuñar el concepto de "cultura de la violencia", como resultado de rasgos culturales de esencia nacional. Esta tendencia nacional a la solución violenta de los conflictos tendría raíces históricas de largo plazo, que se remontan a los enfrentamientos entre Chibchas y Caribes, las guerras internas de los Muiscas y las guerras de la conquista española, pero que se refuerzan con la Revolución de los Comuneros, las guerras de la Independencia, los conflictos internos denominados como la Patria Boba, los enfrentamientos entre realistas y patriotas, y las nueve guerras civiles de carácter nacional del siglo XIX, amén de otros setenta enfrentamientos de orden local y regional.

La historia muestra que los intentos de construcción de una sociedad liberal en Colombia han fracasado. Las frustraciones son numerosas, "todas las promesas liberales que se concretaron ampliamente en los países avanzados, se incumplieron sistemáticamente en el caso colombiano" (Kalmanoviitz y Caro, 2000, p.18). Y de manera más enfática afirma: "el liberalismo clásico nunca tuvo una verdadera oportunidad en la historia del país". Desde el punto de vista de la exclusión, Marco Palacios (2001) afirma:

(...) el continuismo colombiano ha generado en las clases dirigentes $y$ en las clases medias prósperas y correctamente educadas una mentalidad excluyente, una especie de mentalidad neo-apartheid que encuentra su razón de ser en la exclusión y segregación implícitas en el modelo de economía política. (p.18).

Con el gobierno de Andrés Pastrana Arango terminó políticamente el siglo XX. Su promesa de "Cambio para construir la paz", nunca se concretó, ya que la solución política negociada del conflicto armado interno no encontró el camino y esto es porque las partes son radicales en sus posiciones para negociar, empezando por la posición del gobierno colombiano que siempre ha defendido los intereses de la oligarquía dominante sin querer ceder nada significativo.

Al igual que en las anteriores campañas, el conflicto armado y la búsqueda de una solución, estuvieron en el centro de los programas de los distintos candidatos presidenciales. Desde distintos ángulos, las nuevas fórmulas de tratamiento al problema indicaban críticas contundentes al fracasado experimento de negociación de la administración de Pastrana Arango con las FARC.

En el gobierno de Uribe Vélez, se planteó la llamada "seguridad democrática" que se resume en la filosofía militarista heredada de Busch, la guerra contra el terrorismo que buscaba reafirmar el colonialismo unilateral de los Estados Unidos como policía del mundo y Uribe erigiéndose como un simple peón del imperio que invirtió la mayor parte del presupuesto nacional en guerra y por su ansia de permanecer en el poder a como diera lugar, fomentó la corrupción, la 
pobreza y el desempleo, privatizó un sin número de instituciones públicas hasta el punto de conseguir la reelección, cambiar las reglas de juego de la Constitución del 91 y haciéndose reelegir por la modalidad del cohecho donde se compró el voto de Teodolindo Avendaño y Yidis Medina en el Congreso. Su gobierno estuvo implicado con la presencia del paramilitarismo no sólo en el Congreso (estimada en un $30 \%$ ), sino en otras dependencias de la administración pública como alcaldías y gobernaciones. En el gobierno de Uribe, proliferaron los llamados "falsos positivos" (ejecuciones extrajudiciales), donde siempre están implicados miembros de las fuerzas militares, delitos considerados de lesa humanidad, se dieron masacres y chuzadas a magistrados de la Corte Suprema y a políticos de izquierda y, aunque aparentemente disminuyó el accionar de la guerrilla, sin embargo, se descubrió que negociaron en cabeza de su Comisionado de Paz, Luis Carlos Restrepo, falsas desmovilizaciones, como en el caso del llamado Frente Cacique Gaitana de las FARC, en lo cual se descubrió que fue otro de los tantos falsos positivos y de igual forma se dieron estas falsas desmovilizaciones del lado del bando de los paramilitares, que a pesar de la extradición de los comandantes de frente, lo que se critica por poder llegar a ser un impedimento para la verdadera "reparación" de las víctimas; sin embargo, hoy se ve que las bandas criminales del paramilitarismo lo único que hicieron fue cambiar de nombre, porque ahora realizan las mismas actividades criminales con el nombre de las llamadas BACRIM.

Otros casos vergonzosos del gobierno de Uribe Vélez, fueron los de su ministro de agricultura, Andrés Felipe Arias con su planteamiento de que las 17.000 hectáreas de la finca Carimagua, no debían ser entregadas a desplazados por la violencia sino a un reducido grupo de empresarios, que podían hacer inversiones rentables, en plantaciones de caucho y palma. $Y$ es que la filosofía que maneja Arias es que para erradicar la pobreza hay que dar a los ricos más plata de la que ya tienen. El caso de las zonas francas donde están incluso, implicados los hijos de Uribe, Jerónimo y Gabriel, en el terreno que compraron en Mosquera avalado por las firmas de sus ministros y la suya propia por la suma de 33.000.000 millones de pesos, en el transcurso de una semana o un mes se valorizó en algo así como en 5.000 .000 millones de pesos. Aparece nuevamente el señor Andrés Felipe Arias, hoy preso y cobijado con medida de aseguramiento, con el caso de Agro Ingreso Seguro, en donde se roba del erario público de los colombianos más de 6.000 .000 millones de pesos para entregárselos a los ricos del país, sobre todo en la Costa Atlántica y dejándole a los campesinos pobres que debían ser los verdaderos destinatarios de esos recursos, cheques que al parecer oscilaban entre 5.000 y 50.000 pesos y si sobrepasaron esta suma, no fue nada significativo en comparación a los ingentes recursos que entregó a las familias ricas del país. $Y$ es que según las investigaciones que todavía están en curso fue a cambio de que apoyaran su potencial candidatura a la presidencia de la República que por fortuna no se dio. Lo más curioso es que Uribe dice ser el único inmaculado en toda esa olla de corrupción y según él mismo, nunca se enteró de nada, ni jamás de los jamases supo, ni sabe nada de corrupción y delitos públicos que se dieron en su gobierno, igual que en el caso del reparto de las notarías relacionadas con su reelección, para no hablar más de esta pesadilla.

Ahora, Juan Manuel Santos, llegó con la bandera de la llamada unidad nacional cuyo propósito no parece ser otro que el mismo que tuvo el gobierno del Frente Nacional a principios de la segunda mitad del siglo XX, cual fue, acabar con todo lo que se hiciera llamar 
oposición y continuar con el mismo estilo de gobierno unidimensional que siempre se ha impuesto aunque sea con diferentes banderas.

\section{Conclusión}

Para finalizar no se puede dejar de mencionar la matanza del Palacio de Justicia en la toma del M-19, donde no se respetó la vida de los magistrados de la Corte Suprema de Justicia, ni de los mismos militantes de dicho grupo alzado en armas, como tampoco la de los civiles que allí se encontraban en 1985, donde se ha podido constatar que hubo ejecuciones extrajudiciales; caso que todavía reposa en la impunidad; como también, el del genocidio del movimiento político izquierdista de la Unión Patriótica, cuyos militantes fueron vilmente exterminados después de haberse desmovilizado para entrar en la lucha política fuera de la lucha armada; dicho genocidio también está impune.

Los diferentes cambios socio-políticos que enfrentó el país, a través de su historia, han marcado la ruta o el mapa por donde se ha desarrollado la democracia, es, por ende, que cuando se explica la democracia consociativa se identifica con lo que sucedió en el periodo de colación, pero que, a la vez, se observa que ocurre en la actualidad, al generarse en cada campaña política estrategias de coalición y de cooptación política, sin estimar que dichas prácticas debilitan a una democracia y la fracturan, pues como se ha observado en aras de sostener una hegemonía política se consolidan caminos que no buscan el bien común sino los medios para sostener y mantener el poder político.

\section{Referencias}

Ayala, C. (1996). Resistencia y Oposición al establecimiento del Frente Nacional. Bogotá, Colombia: Universidad Nacional de Colombia.

Araoz, S. (1977). Historia del Frente Nacional y otros ensayos. Bogotá, Colombia: Presencia.

Arendt, H. (1993). ¿Qué es la Política? Barcelona, España: Paidos.

Bedoya B. (1988). La muerte del frente nacional: historia secreta y pública. Santa Fe de Bogotá, Colombia: Graphic Center ed.

Bilbeny, N. (1998). Introducción a la Filosofía Política. Barcelona. Colombia: Ariel.

Bobbio, N. (2006). Estado Gobierno y Sociedad, por una teoría general de la política. trad. José F. Fernández. México: FCE.

Cámara de Representantes (1959). Por qué y cómo se forjó el Frente Nacional. Bogotá, Colombia: Imprenta Nacional.

Corsi O., José C. y González S. (1997). El Estado auténtico, un modelo político de comunión. Bogotá, Colombia: Senado de la República.

Galvis N. (2008). El Frente Nacional (Institución Política en Colombia). Bogotá, Colombia: FCE

Galvis, S. y Donadio, A. (1986). Colombia Nazi, 19391945. Bogotá, Colombia: Planeta.

Hartlyn, Jonathan (1993). La política del Régimen de coalición: La experiencia del Frente Nacional en Colombia. Bogotá: Centro de Estudios Internacionales de la Universidad de los Andes (CEI) - Ediciones Uniandes - Tercer Mundo Editores.

Jaramillo, U. (1962). Rousseau y el pensamiento colombiano en los siglos XVIII y XIX. En la obra Presencia de Rousseau. México: UNAM.

Kalmanoviitz, S. y Caro, M. (2000). El Banco Nacional y el Estudio [Mimeo]. Bogotá, Colombia: Banco de La República.

Lijphart, A. (1989). Democracia en las sociedades plurales una investigación comparativa. Trad. de Susana Serdán Vázquez. Bueno Aires: Grupo Editor Latinoamericano.

Lleras A. (1962). El primer gobierno del frente nacional. Bogotá, Colombia: Imprenta Nacional.

Molina, G. (1974). Las ideas liberales en Colombia $16^{a}$ ed.) T.III de 1953 a la iniciación del Frente Nacional. Bogotá, Colombia: Tercer Mundo. 
Nisbet, R. (1995). Conservadurismo. Madrid: Alianza.

Pacheco B. R. (2001). Historia Constitucional Colombiana. Bogotá, Colombia: USTA.

Palacios, M. (2001). De Populistas, mandarines y violencias. Luchas por el poder. Bogotá, Colombia: Planeta.

Pécaut, D. (1989). Crónica de dos décadas de política colombiana 1966 - 1988. Trad. de Jorge Orlando Melo profesor universidad nacional. Bogotá. Colombia: Siglo veintiuno editores.

Pombo, M. y Guerra J. (1953). Constituciones de Colombia. Bogotá, Colombia: Ministerio de Educación Nacional.
Ramírez C. (2001). El Estado de justicia; más allá del Estado de derecho. Bogotá, Colombia: El Buho.

Reale, G. y Antiser,i D. (1988). Historia del pensamiento filosófico y científico. Versión castellana de Juan Andrés Iglesias. Barcelona, España: Herder.

Romero, J. (1987). Estudio de la mentalidad burguesa. Madrid, España: Alianza.

Strauss L. (1970). Qué es Filosofía Política. Trad. Armado A. de la Cruz. Madrid: Guadarrama.

Tirado M.(1989). Nueva Historia de Colombia, Bogotá: Planeta.

Vargas, C.(1992). Historia crítica del Frente Nacional. Santa Fe de Bogotá, Colombia: Foro Nacional por Colombia. 
